\title{
iDeepMon - intelligent deep mine shaft inspection and monitoring
}

\author{
N Benecke DMT GmbH \& Co. KG, Germany
}

\begin{abstract}
This paper initially presents the existing solutions in shaft surveying and monitoring, in particular, kinematic survey systems. Shaft surveying and monitoring is essential to the success of high-performance underground mining operations and to enable 24 hour/seven day shaft operability. Therefore, mining companies have a serious interest in cost-efficient, time-efficient and safe technologies to monitor, inspect and maintain their shafts. In addition, mining companies are obliged to carry out monitoring and inspection of their shafts as a mandatory part of the operational permissions by mining authorities.

Due to this demand, the iDeepMon project was awarded funding from the European EIT RawMaterials Network. The project is under way to enhance the already existing shaft surveying technologies towards a fully automated process of shaft inspection, which will be integrated into the overall control process of an autonomous mine.
\end{abstract}

The main part of the paper will provide information about the iDeepMon project, project achievements, and the results and benefits for shaft operations.

Keywords: automated shaft surveying, monitoring, inspection

\section{$1 \quad$ Existing shaft surveying systems}

As previously reported (Benecke et al. 2016), shaft inspection and monitoring is important for any shaft in operation, as well as for abandoned shafts for various purposes, such as regular inspection of changes and damage, monitoring whilst mining activities are impacting the shaft or as a basis for repair work, and reconstruction work or shaft closure work. In all cases, diverse information is needed, e.g. detailed documentation and condition of guides, walls and installations, inclination, deformation and damage of shaft walls, and plumbing of coordinates to deeper mine levels.

It is important to acquire this information, however, acquisition is difficult due to specific and dangerous conditions for any survey in a mineshaft, e.g. risks due to working at heights, environmental factors such as dust, water, possible extreme temperatures, poor illumination, and limited space and time windows for surveying.

Traditional survey systems for shafts are not very efficient in terms of safety, speed and accuracy. The main requirements for an efficient system are:

- Safety: mostly remote operation, no personnel entry to dangerous locations in the shaft.

- Safety for coal mines: System must fulfil safety requirements concerning presence of methane gas.

- Speed: Measurement duration (equivalent to operation downtime) less than one shift per shaft is requested.

- Accuracy: Millimetre in local details, approx. $10-20 \mathrm{~cm}$ in absolute coordinates.

Therefore, two new innovative shaft survey systems have been developed within recent years; one for shafts with hoisting facilities (Section 1.1) and one for shafts without hoisting facilities (Section 1.2). Both systems have already been presented in detail (Benecke \& Weber 2013).

This paper was originally published in the Proceedings of the XVI International Congress for Mine Surveying. Acknowledgement is given to the Australian Institute of Mine Surveyors for their permission to republish this paper. 


\subsection{Cage-based system}

The main idea behind this system is the use of kinematic laser scanning technology, already known from street or rail mapping, but in a vertical downward direction in deep mining shafts. However, this is not considered an easy approach, and made for a challenging research and development project. In particular, because of the missing availability of Global Navigation Satellite System (GNSS) data, the specific environmental conditions in mining shafts (e.g. dust, humidity, dirt, methane gas in coal mines) and strict safety regulations.

The system layout consists of the following:

- Installation of an inertial measurement unit (IMU) and two laser scanners (IMAGER 5006i) on a hoisting cage; if requested as ex-proofed version.

- Kinematic survey of the shaft with both technologies:

- Measurement of absolutes of shaft position by an IMU.

- Simultaneous data logging (shaft walls and installations) of both scanners in profiler mode.

- Odometer for depth control.

- Internal power supply and data storage.

- Control unit for synchronisation purposes.

The system was developed by DMT GmbH \& Co. KG (DMT) and tested on several of RAG Deutsche Steinkohle AG, Germany's coal mines. Depending on client requirements, several different results can be evaluated:

- Point cloud visualisation in 3D and unwrapping.

- Deformation analysis.

- Cross-sections.

- Computer-aided design (CAD) modelling of installations.

- Detailed measurement of position and distances of guide rails.

- Absolute positioning track.

The main features of the system include the following:

- Three-dimensional digital object documentation by kinematic laser scanning while cage driving.

- Remote and complete 3D documentation of shaft walls and installations (e.g. guide rails, pipelines or spreaders) with damage and anomalies.

- Absolute positioning and deviation from vertical by inertial measurement technology.

- Fast, easy and complete data acquisition independent from lighting or surface conditions.

- Safe execution of survey without exposure of personnel to dangerous shaft work.

- Compliance with EU ATEX regulations, the worldwide highest standard concerning the use of electrical devices in explosive atmospheric conditions.

- Highest reliability and accuracy:

- Absolute accuracy (for depth of 1,000 m): $10 \mathrm{~cm}$.

- Relative accuracy of laser scanner measurement: $1 \mathrm{~mm}$ at $10 \mathrm{~m}$ distance.

- Various options for data visualisation and analysis (e.g. 3D, virtual reality, video and CAD). 


\subsection{Wireline system}

In Germany in particular, a large number of abandoned shafts are not backfilled for several reasons, e.g. water pumping. Also, a significant number of old mine shafts (more than 10,000 in total, with some older than 100 years) exist, and are becoming more and more likely to cause surface damage in form of sinkholes.

DMT was asked by clients if there was an opportunity to scan these old mine shafts, where typically no cage is installed. After some trial testing, DMT developed the wireline-shaft-survey-system within the framework of a European Research Project (MISSTER 2012).

The wireline system consists of the following main components:

- 2D laser scanner (profiler).

- Inertial measurement unit.

- $360^{\circ}$ RGB camera.

- Sensor for barrier identification.

- Gas sensors $\left(\mathrm{O}_{2}\right.$ and $\left.\mathrm{CH}_{4}\right)$.

- Power supply.

- Data processor and storage.

Even though the system layout is different from the cage-based system, the results and options for data evaluation and visualisation are more or less the same. Figure 1 illustrates the high quality of the acquired data from a tubbing shaft.

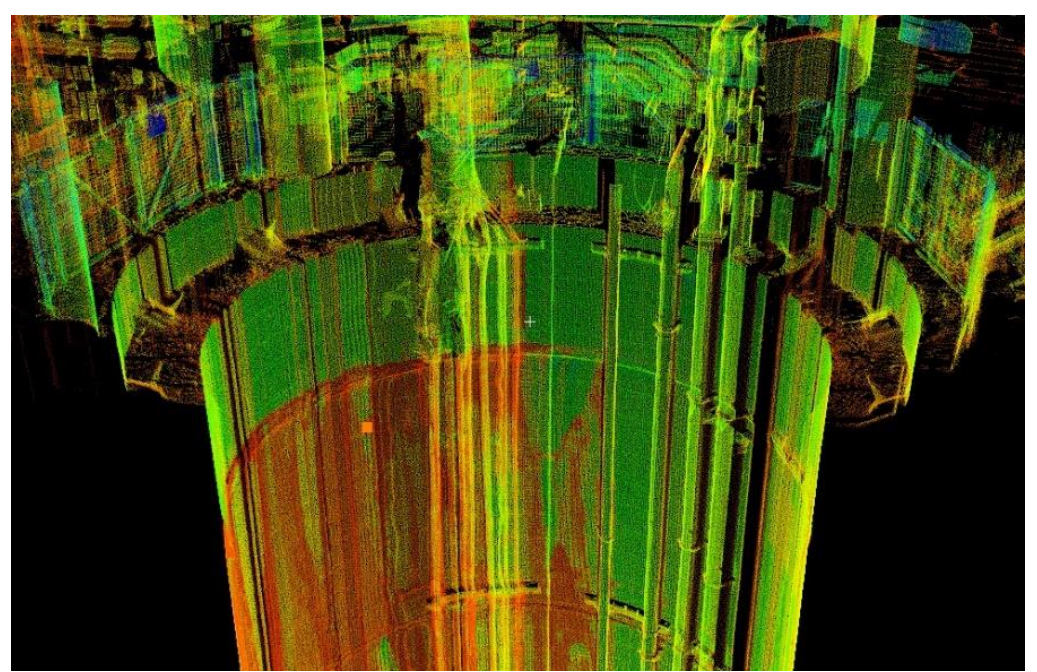

Figure 1 3D point cloud of a shaft

The achievable reliability and accuracy of the measurements with the wireline-shaft-survey-system is the best available quality of all existing systems for surveying of shafts without installations:

- Absolute accuracy (for depth of $1,000 \mathrm{~m}$ ): 20 to $30 \mathrm{~cm}$.

- Relative accuracy of laser scanner measurement: $1 \mathrm{~cm}$ at $10 \mathrm{~m}$ distance.

\subsection{Practice example for shaft surveying}

With both operational shaft surveying systems, DMT has successfully carried out more than 100 operational projects in Europe, Russia and Australia (Benecke et al. 2016) within the last five years. Several projects are now monitoring projects, where we get a time series of changes over time from repeated surveys (Figure $2(a)$ and $(b)$ ). 


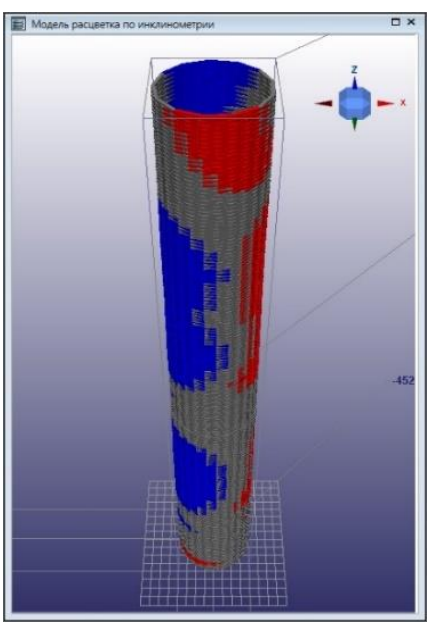

(a)

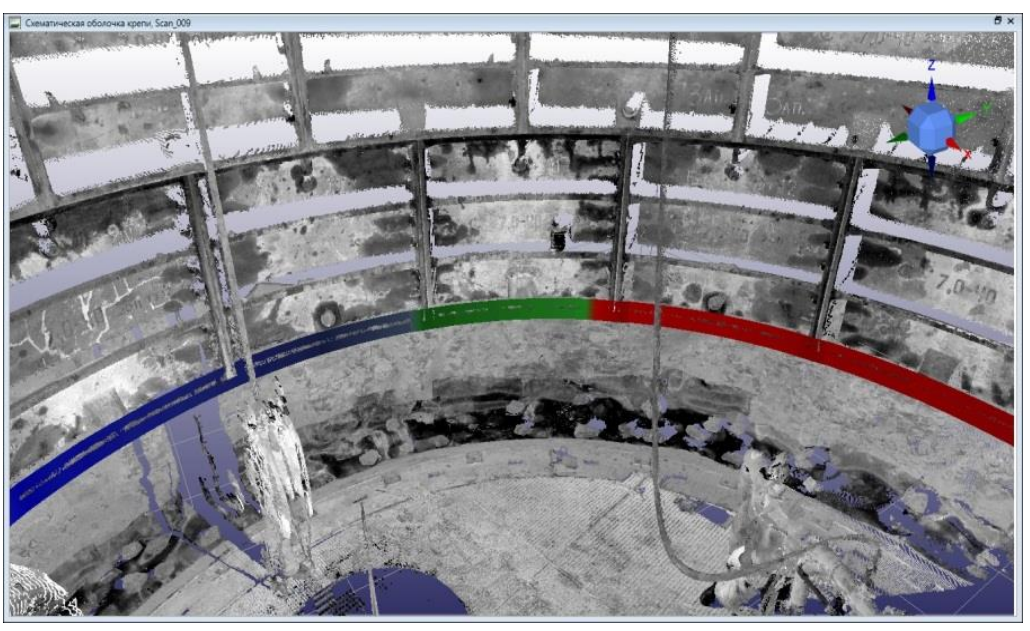

(b)

Figure 2 (a) Differences in shape against drawings; and, (b) Overbreak/underbreak model

\section{$2 \quad$ From surveying to inspection}

The presented system for kinematic shaft survey is an important step forward in quality, reliability, safety and effectiveness in shaft surveying. Further developments are planned or already under development. In particular, the use of high-resolution video camera systems, in addition to laser scanners, will offer new opportunities in data quality and evaluation.

The ongoing developments in this area are important steps toward the vision of an automated shaft inspection system, which can easily be installed by the shaft engineer and may in future replace the regular visual shaft inspections. The system will record the data with a high level of quality and will relay it in real time to the mine control room, where an intelligent algorithm will automatically identify changes in comparison to former inspections. However, shaft inspectors can do their work on a computer or in a 3D theatre in a comfortable and safe environment, and perform data evaluation and impact investigation on the basis of reliable and comparable datasets. The following subsection will give an overview of the most important development project, iDeepMon (Section 2.1), as well as further projects, named PILOT3D and UNDROMEDA, which deliver additional value for the different required shaft inspection tasks.

\section{$2.1 \quad$ iDeepMon - intelligent deep mine shaft monitoring}

iDeepMon is a so-called 'upscaling project' within the EU-funded framework of EIT RawMaterials. More details are available at the project webpage (Benecke \& Koch 2016).

EIT RawMaterials is a strong European knowledge and innovation community with more than 120 partners from industry, research and education (Hanghøj 2016). The vision of EIT RawMaterials is to boost the competitiveness, growth and attractiveness of the European raw materials sector via radical innovation and entrepreneurship.

The main objective of iDeepMon is the further development of existing shaft surveying systems towards an automated and remotely controlled shaft inspection system which allows an advanced maintenance approach for deep mine shafts with automatic tracking of the shaft degradation process. The project concept contributes to the strategic objectives of EIT RawMaterials, i.e. improving the safety of operations, in particular mining automation, remote control and advanced maintenance systems.

\subsection{1 iDeepMon - feasibility study}

As the first step of the project, an online user survey was carried out and has been sent to approximately 60 people in companies that are operating deep mine shafts. A total of 19 completed questionnaires were received, which is a response rate of $31.6 \%$. Most answers came from Europe (including former Soviet 
Union countries) with 11 responses, three from South Africa, three from Australia and two of unknown regional origin. These answers are representing approximately 100 mines with up to 200 shafts with hoisting facilities and another 140 shafts without hoisting facilities (Kröger \& Benecke 2016).

One of the main findings of the questionnaire is that almost all deep mine shafts have to be monitored due to operational issues and mandatory regulations.

At present, this shaft inspection is mainly done by visual methods plus traditional surveys (plumbing plus total stations) for specific purposes (e.g. in case of deformations). These inspections are time consuming, with downtime and production losses, are dangerous for the executing staff, and deliver low quality results. In particular, downtime of a shaft for monitoring will essentially decrease the productivity of a mine. Furthermore, current systems do not permit any simple manner of comparing surveys over time.

However, the mines which are using traditional methods need a very low investment in technology and knowhow. In contrast, using the proposed innovation needs more investment in technology and additional training of staff. But, with the realisation of the planned system, the user can expect to receive several benefits.

The reduction of factors encouraging human error, such as fatigue, loss of concentration and poor vision, is one of the most important required enhancements. This is directly linked to a higher level of accuracy, quality and reliability of the collected data. The user also expects to receive a first, mostly automatic data evaluation nearly in real time. Further detailed investigations, with a detailed 3D shaft model and early detection of changes and defects, will become possible in a short timeframe within the comfort of the office.

The registered data from each inspection drive are an entire log of the shaft, which can easily be used for inspections, renovations and modifications. In particular, the user expects a serious increase in the level of safety.

Additionally, the following benefits are expected by the user:

- Virtual marking of locations within a shaft which require further attention and then the automatic collection of more detailed information at those locations in future inspections.

- Allowing the generation of history reports, to record the development of features at specific locations in the shaft to simplify the monitoring of critical changes over time.

- Minimising downtime for monitoring, thus increasing production time.

- Avoiding additional downtime due to mining inspectorate order after their official inspections.

- Availability of reliable documentation of inspection results in any case of damage/accidents.

All these advantages will hopefully sum up to a noteworthy decrease in operating costs and to a sufficient increase of transport capacity of the shaft. From discussions with international mining companies from Canada, South Africa, Russia and Belarus, we received information that the financial benefit can amount of hundreds of thousands of euros per year at minimum, and up to some million euros at maximum, depending on the type of mined mineral and particular requirements on inspection (frequency, intensity, etc.). In particular, in cases where the early detection of defects helped to avoid accidents or interruption of production, the return on investment will be achieved much quicker. For example, the value of the production of an Australian ore mine is between EUR 2 and $5 \mathrm{M}$ per day (depending on commodity prices and several other influencing figures). However, it is obvious that just a one week production stop due to the unavailability of the main shaft will dramatically endanger the profitability of a mine.

The final developed system, when achieving technical readiness level nine, is planned as a permanent system on a mine site, operated by the mining company. Today, two options are possible; one permanently fixed system for each shaft and one system for one mine (with some shafts), which can easily be moved from one shaft to another within a regular schedule. The system may be operated by people from the mining company or by external service providers. 


\subsection{2 iDeepMon - current status}

After finalising the feasibility study at the end of 2016, the project team began the development of the new automated inspection system, iDeepMon, with selection of components, i.e. first testing the selected components in a laboratory and a real underground test mine (Figure 3), hardware design and initial software developments. It is planned to have the prototype system developed by the end of 2017, in order to carry out first practice tests in a real mine environment in early 2018 at Boliden's Kristineberg Mine, Sweden. From the project schedule, it is planned to have the tested prototype ready in mid-2018.
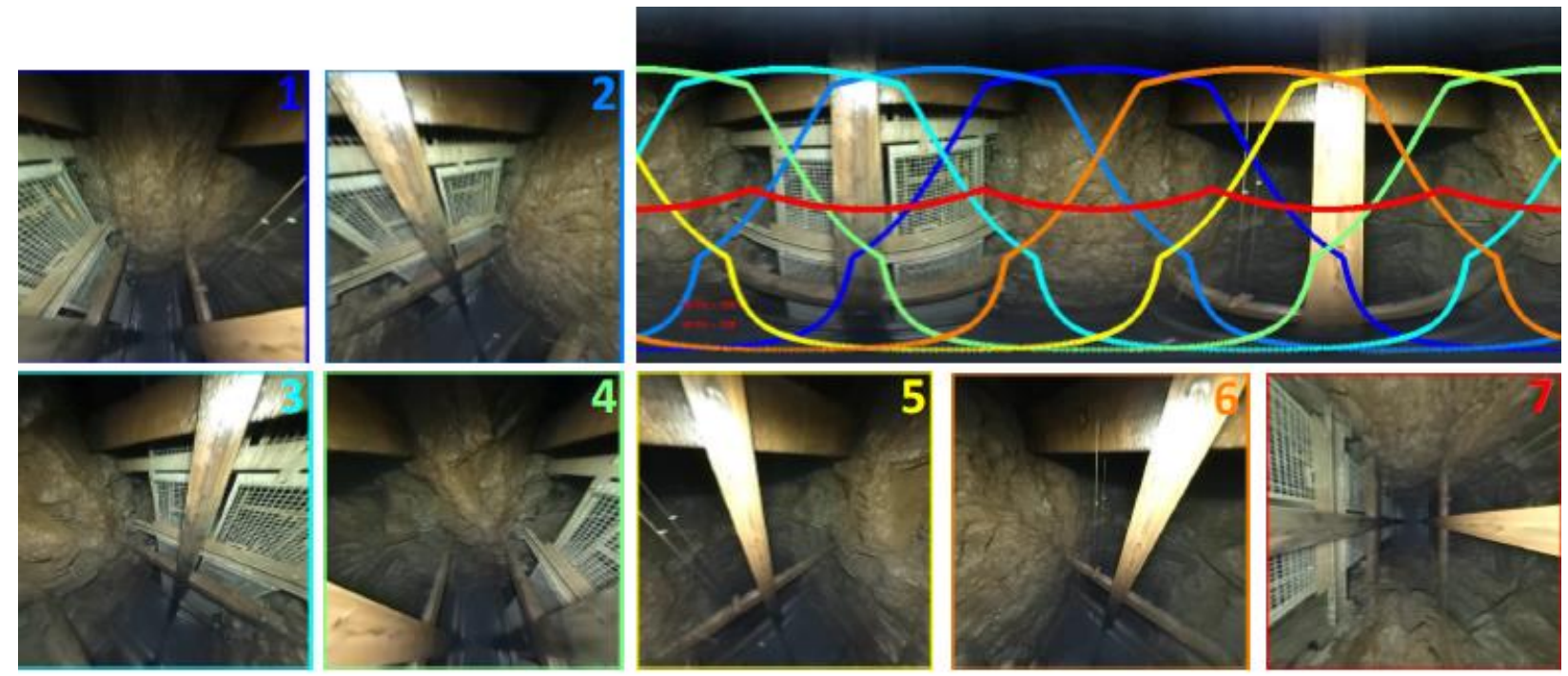

Figure 3 Images of first component tests at Shaft Reiche Zeche

The project team consists of the following organisations:

- DMT, Germany as project leader, hardware developer, main prototype testing organisation and system facilitator.

- German Aerospace Center (DLR, a governmental research institute for space technologies) as developer of the main integrated sensor technology and algorithm, named 'Integrated Positioning System' (IPS).

- Technical University Freiberg (TUBAF), Germany as scientific sensor system and software developer with underground testing facility 'Reiche Zeche'.

- Mining University Leoben (MUL), Austria as scientific data processing and data storage developer.

- Technical University Nuremberg (THN), Germany as scientific data evaluation software developer.

- Technical University Gliwicé (SUT), Poland as shaft expert evaluators.

- New Boliden AB (Boliden), Sweden as user partner and pilot testing organisation.

\subsection{Additional relevant developments}

\subsubsection{Integrated Positioning System}

IPS is an optical sensor-based development by DLR for ego motion estimation without any prior knowledge of the environment. It is able to operate in outdoor spaces as well as indoors, e.g. buildings and tunnels.

The system is based on a multi-sensor approach. Firstly, the aforementioned optical sensor is used as the primary sensor. Its data can be combined with measurements of a low-cost IMU and optionally by a tilt sensor. As each sensor has different error characteristics and error behaviour, the advantage of this combined approach is the capability of the system to compensate the error-proneness of each single 
sensor, extending the system's robustness significantly. The main purpose of IPS in this state is the development of visual navigation methods and technologies to determine the user's position and orientation (Born et al. 2016), therefore this technology is the basis for the iDeepMon system.

\subsubsection{PILOT3D inspection system}

In further developments, the system has been extended by additional sensors and functionalities, opening the research platform to different applications where indoor navigation becomes crucial (Gödde et al. 2017). Today, DMT, as business partner to facilitate products based on IPS, has brought the handheld device, PILOT3D, as the first product to the market (Figure 4).

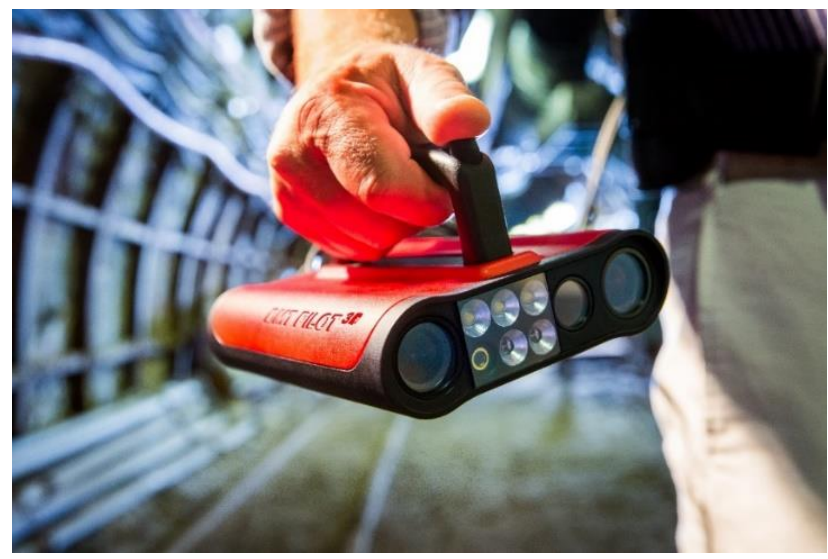

Figure 4 Handheld inspection tool, PILOT3D

The integrated positioning system, PILOT3D, allows precise and real-time navigation without previous knowledge of the neighbourhood or special referencing (e.g. GNSS). It is the optimal solution for enclosed environments such as underground facilities. In addition, the IPS technology will be integrated in the iDeepMon shaft inspection system to guarantee the highest quality and reliability for the position of the mineshafts in any depth.

\subsubsection{UNDROMEDA}

The new UNDROMEDA project (starting in 2018) aims to develop a robotic underground measurement system for autonomous 3D mapping and monitoring (Zimmermann 2017). The system is based on a mobile wheel-driven platform, which additionally carries a flying drone to approach particularly unknown, difficult to access or hazardous areas in underground mines and further underground environments, such as tunnels or sewer systems (Figure 5). For this, it is necessary to integrate advanced positioning, navigation and mapping sensors such as laser scanning, radar, inertial technology as well as innovative algorithms such as laser simultaneous localisation and mapping (SLAM), IPS optical navigation and virtual reality control.

UNDROMEDA is a milestone project within the current leapfrog developments toward the 'invisible, zero-impact, intelligent, safe and fully autonomous mine', and enables one to face the related challenges of future mining concerning social and environmental acceptance, as well as economic efficiency. The autonomous platform and drone will significantly reduce the risk to underground personnel by replacing manual measurements. Automation will reduce time and costs for mapping and monitoring, while advanced sensors and their integration will dramatically enhance the information density and quality. The team of highly experienced project partners will integrate and further develop several existing partial solutions to an innovative high-performance system with capabilities for efficient monitoring, evaluation and detection applications.

While the iDeepMon solution will solve the inspection requirements for shafts with hoisting installations, the UNDROMEDA system will solve the inspection requirements for shafts without hoisting installations, in particular, inclined orepasses. 


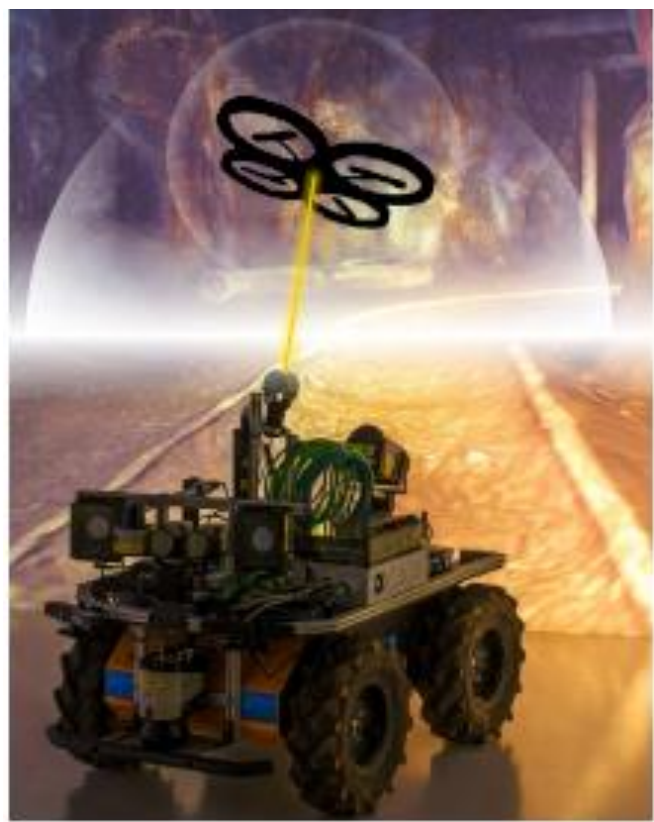

Figure 5 First sketch of UNDROMEDA robotic vehicle

\section{Conclusion}

For high-performance underground mining operations, 24 hour/seven day operability of the shafts is essential to the success of the mining operation in terms of cost, performance, efficiency and safety. Therefore, mining companies have a serious interest in cost-efficient, time-efficient, and safe technologies to monitor, inspect and maintain their shafts. In addition, mining companies are obliged to carry out monitoring and control of their shafts as a mandatory part of the operational permissions by mining authorities. The presented technology developments, both already achieved as well as under construction, are important steps toward the 'invisible, zero-impact, intelligent, safe and fully autonomous mine' of the future.

\section{Acknowledgement}

The author thanks the entire iDeepMon team for their project work and support of this presentation. The author also thanks the European Union, in particular the European Institute for Innovation and Technology (EIT), for their generous funding and reliable support of the project.

\section{References}

Benecke, N \& Weber, M 2013, 'A new approach for shaft monitoring by kinematic laser scanning', Proceedings of the XV Congress of the International Society of Mine Surveying, Institute of Mine Surveyors of South Africa, Marshalltown.

Benecke, N \& Koch, M 2016, EIT RawMaterials Upscaling Project iDeepMon, DMT GmbH \& Co. KG, Essen, viewed 4 July 2017, http://www.ideepmon.eu

Benecke, N, Hancock, P \& Weber, M 2016, 'Latest developments for practice in shaft inspection', Proceedings of the XVI Congress of the International Society of Mine Surveying, Australian Institute of Mine Surveyors, Singleton.

Born, A, Benecke, N, Boerner, A, Rapp, S, Stelzer, P, Tsirigotis, N, Weber, M \& Zuev, S 2016, 'Mobile solution for positioning, 3D-mapping and inspection in underground mining', Proceedings of the XVI Congress of the International Society of Mine Surveying, International Society of Mine Surveyors, published by AIMS (Australian Institute of Mine Surveyors), Singleton.

Gödde, W, Benecke, N, Born, A, Schischmanov, A \& Zuev, S 2017, Intelligent Sensing and Navigation in Mining Automation, oral presentation given at German Day 2017 at the Prospectors \& Developers Association of Canada 2017, Toronto.

Hanghøj, K 2016, 'EIT RawMaterials, a European knowledge and innovation community in raw materials', keynote speech at the First International Conference Mining in Europe: Aachen International Mining Symposia (AIMS) 2016, Institute of Mineral Resources Engineering, RWTH Aachen University, Aachen.

Kröger, G \& Benecke, N 2016, iDeepMon Feasibility Study Report, EIT RawMaterials GmbH, Berlin (not published).

MISSTER 2012, Mine Shafts: Improving Security and New Tools for the Evaluation of Risks, European RFSC Research Project.

Zimmermann, K, 2017, UNDROMEDA - Underground Robotic System for Monitoring, Evaluation and Detection Applications, confidential proposal for EIT RawMaterials call for projects 2017 (not published). 